\title{
ARTICLE OPEN \\ Successful application of prime and pull strategy for a therapeutic HSV vaccine
}

\author{
David I. Bernstein ${ }^{1}$, Rhonda D. Cardin², Fernando J. Bravo ${ }^{1}$, Sita Awasthi ${ }^{3}$, Peiwen Lu , Derek A. Pullum ${ }^{1}$, David A. Dixon ${ }^{1}$,
} Akiko Iwasaki $\mathbb{D}^{4,5}$ and Harvey M. Friedman ${ }^{3}$

\begin{abstract}
One promising approach for a herpes simplex virus vaccine uses a vaccine to prime and a chemoattractant to pull immune cells into the genital tract. We evaluated subunit vaccines (prime) and imiquimod (pull) in the guinea pig (gp) model of recurrent Herpes simplex virus type-2 (HSV-2). Following vaginal HSV-2 infection, gps were vaccinated with various combination of glycoproteins and adjuvant with or without subcutaneous or local applications of imiquimod after infection. Animals were examined daily for recurrent lesions and vaginal swabs collected for recurrent shedding. Although both the vaccines alone and imiquimod alone reduced recurrent HSV disease, the combination of local imiquimod and vaccine (Prime and Pull) was the most effective. In the first study, immunization with the trivalent vaccine alone or imiquimod alone decreased recurrent disease. However, the largest decrease was with the combination of vaccine and local imiquimod ( $P<0.001$ vs. placebo or vaccine alone). No effect on recurrent shedding was observed. In the second study, recurrent disease scores were similar in the PBS control group and the trivalentimmunized group treated with subcutaneous imiquimod however, significant reductions with glycoprotein vaccines and local imiquimod ( $p<0.01$ vs. placebo) were noted. The number of qPCR-positive recurrent swabs, ranged from 5 to $11 \%$ in the vaccinated+local imiquimod groups compared $29 \%$ in the PBS control group $(P<0.05)$. No recurrent swab samples from vaccinated groups were culture positive. We conclude that the strategy of prime (subunit HSV vaccine) and topical pull (intravaginal/topical imiquimod) decreased recurrent HSV more effectively than vaccine alone.
\end{abstract}

npj Vaccines (2019)4:33; https://doi.org/10.1038/s41541-019-0129-1

\section{INTRODUCTION}

Herpes simplex virus type 2 (HSV-2) is estimated to infect 417 million people globally, with 19.2 million new infections annually. ${ }^{1}$ Almost all infected individuals develop recurrences, either recurrent lesions or even more often recurrent shedding in the absence of lesions. ${ }^{2,3}$ Asymptomatic shedding occurs frequently, $>10 \%$ of days and is believed to be the major cause of transmission. $^{2-4}$ Further, neonatal HSV infections produce a serious disease with high rates of morbidity and even mortality despite current antiviral therapies. ${ }^{5,6}$ Genital HSV infections are also recognized to increase the acquisition of HIV and result in increased shedding of HIV. ${ }^{7}$

Available antiviral therapies are somewhat effective in the treatment of recurrent HSV disease; shortening lesion duration and decreasing viral shedding but are only effective during the time of drug administration. 89 Therefore, there has been great interest in developing therapeutic vaccines designed to decrease lesion rates as well as shedding rates. ${ }^{10}$ The evaluation of immunotherapy for recurrent herpesvirus infection dates back to at least the 1930s. However, the therapeutic potential of immunotherapy was not demonstrated conclusively until 1988, when Stanberry et al. ${ }^{11}$ were able to show, using the guinea pig model of genital herpes, that a vaccine could indeed reduce the number of genital herpes recurrences. This preclinical model was later extended to show the effects of immunization on recurrent shedding. ${ }^{12}$ Most recently, this preclinical work was supported by several clinical trials ${ }^{13,14}$ of a bivalent HSV-2 vaccine provided with a potent adjuvant. In these trials, vaccination reduced recurrent lesions or recurrent shedding rates by $\sim 50 \%$.

We sought to improve upon this rate by employing a prime and pull strategy. ${ }^{15}$ The prime and pull strategy relies on two steps: (1) conventional parenteral vaccination to elicit systemic T-cell responses (prime), followed by 2 ) recruitment of activated T cells via topical administration of a $\mathrm{T}$ cell attractant (pull), where such $T$ cells establish long-term protective immunity. The original description applied to prophylactic HSV immunization and showed the primary CD8 T-cell responses were of similar magnitudes in the spleen, whereas the frequency and number CD8 T cells in the vagina were significantly higher in vaccinated mice treated with a vaccine and the chemokine pull as compared with the control immunized mice without the "pull". Furthermore, the action of the chemokine pull was restricted to the genital mucosa, as CD8 T-cell recruitment to the vagina-draining lymph nodes was limited. Importantly, the prime and pull strategy conferred near complete protection against primary challenge with genital HSV-2 infection compared with prime alone. ${ }^{15}$ In this manuscript we extend the application to therapeutic vaccines and show that the frequency of recurrent disease and recurrent vaginal shedding was reduced most effectively by the combination of prime (glycoprotein vaccine) and pull (vaginal imiquimod).

\footnotetext{
${ }^{1}$ Department of Pediatrics, University of Cincinnati College of Medicine, Cincinnati, OH, USA; ${ }^{2}$ School of Veterinary Medicine, Louisiana State University, Baton Rouge, LA, USA; ${ }^{3}$ Infectious Disease Division, Department of Medicine, Perelman School of Medicine, University of Pennsylvania, Philadelphia, PA 19104-6073, USA; ${ }^{4}$ Department of Immunobiology, Yale University, New Haven, CT, USA and ${ }^{5}$ Howard Hughes Medical Institute, Chevy Chase, MD, USA

Correspondence: David I. Bernstein (david.bernstein@cchmc.org) or Akiko Iwasaki (akiko.iwasaki@yale.edu) or Harvey M. Friedman (hfriedma@pennmedicine.upenn.edu)
}

Received: 4 March 2019 Accepted: 24 June 2019

Published online: 01 August 2019 


\section{RESULTS}

Study 1

In study one (Fig. 1), immunization with the trivalent vaccine plus adjuvant decreased recurrent disease scores from $9.9 \pm 4.5$ in the placebo group to $7.8 \pm 5.1$ but the decrease was not significant (Fig. 1b). Treatment with imiquimod (Ivag/topical) alone also decreased recurrences $(5.0 \pm 2.6, P<0.02$ vs. placebo) but the largest decrease was with the combination of vaccine and imiquimod (Ivag/topical). This group had significantly reduced recurrent lesion scores $(1.1 \pm 1.3)$ compared with the placebo group as well as to the vaccine alone group ( $P<0.001$ to each) (Fig. 1b). No evidence of local irritation or inflammation was observed during the treatment with imiquimod. Similarly, after the three vaccinations recurrent lesion scores were significantly lower in the vaccine and imiquimod (Ivag/topical) group compared with the placebo $(P<0.001)$ or vaccine alone group $(p<0.01)$ (Fig. $1 c)$.

No significant differences were found between groups in the number of animals that developed recurrent lesions $(P \geq 0.20$, Fig. 1b) when the entire period of observation was evaluated. However, the number of animals with recurrences was significantly lower $(P<0.02)$ in the $\mathrm{gC} 2 / \mathrm{gD} 2 / \mathrm{gE} 2+$ imiquimod group (Ivag/topical) in both the period after the second dose $(9 / 15,60 \%)$ and after the third dose $(7 / 15,47 \%$, Fig. 1c) when compared to the PBS group (15/15, 100\% for both periods).

An effect on the number of days with recurrent vaginal shedding of HSV-2 DNA was not seen in this experiment (Fig. 2). In fact, the number of days with recurrent shedding was significantly increased in the groups receiving the $\mathrm{gC} 2 / \mathrm{gD} 2 / \mathrm{gE} 2$ vaccine or the $\mathrm{gC} 2 / \mathrm{gD} 2 / \mathrm{gE} 2$ vaccine+imiquimod compared with placebo over the entire period $(P<0.05)$ Fig. 2a. The comparison after the third immunizations shows that shedding was more similar and not significantly different comparing vaccinated groups to placebo (Fig. 2b).

Analysis of the frozen vaginal sections showed the effect of the prime and pull strategy on the number of CD4 but especially CD8 T cells in the vaginal tissues as compared with either the placebo, trivalent vaccine $(\mathrm{gD}+\mathrm{gE}+\mathrm{gC})$ alone or the pull (imiquimod) alone). Thus, although some CD8 T cells were detected in all groups, the animals vaccinated with $\mathrm{gC} 2 / \mathrm{gD} 2 / \mathrm{gE} 2+$ imiquimod Ivag/topical had an increased number of CD8 T cells, particularly within the epithelial layer (Fig. 3a). We also observed an increase in the number of CD4 T cells in the submucosa within the group receiving $\mathrm{gC2} / \mathrm{gD} 2 / \mathrm{gE} 2+$ imiquimod Ivag/topical treatment (Fig. 3b).

\section{Study 2}

In the second experiment we sought to determine if the trivalent vaccine was more effective than the monovalent gD2 or bivalent vaccines and whether parenteral (SQ) imiquimod would be as effective as the local (Ivag/topical) administration, thus providing evidence as to whether the effects were due to the adjuvant activity of imiquimod or the pull of effector cells into the genital tract. All vaccinated groups that were treated Ivag/topically with imiquimod showed a significant decrease $(p<0.01)$ in recurrent disease scores (ranging from 1.2-2.7 $\pm 1.7-2.7$ ) when compared with the control PBS group $(7.7 \pm 5.6)$ or the trivalent vaccine group treated with SQ imiquimod (7.0 \pm 4.4$)$ (Fig. 4). There were no significant differences $(p>0.50)$ compariing groups receiving Ivag/ topical imiquimod+trivalent $(\mathrm{gC} 2+\mathrm{gD} 2+\mathrm{gE} 2)$, bivalent (either $\mathrm{gD} 2+\mathrm{gC2}$, or $\mathrm{gD} 2+\mathrm{gE2}$ ), or monovalent vaccine (gD2) (Fig. $4 \mathrm{a}$, b). Similarly, recurrent lesion scores remained significantly lower $(p<0.05)$ in all the vaccinated groups that were treated Ivag/ topically with imiquimod during the period after the third vaccination (Fig. 4c). No evidence of local irritation was observed during the treatment with imiquimod.

All of the vaccinated groups receiving Ivag/topical imiquimod also had a significant reduction in the percentage of animals with recurrent lesions (ranging from 53 to $67 \%$ ) when compared with the control PBS group (15/15, 100\%, $p<0.05)$ (Fig. 4b). The reduction after all three immunizations (days 43-70) is even greater (Fig. 4c). With the exception of the gD2 \& gE2+CpG+Alum (group 5), all of the groups receiving vaginal imiquimod also had a significant reduction in the percentage of animals with recurrent lesions when compared with the trivalent vaccine groups receiving SQ imiquimod (group $3, p<0.05$ ). Similar to the lesion scores, there were no differences in comparisons made between the groups treated vaginally with imiquimod.

Recurrent shedding was also significantly reduced. The number of positive swab samples was lower in all of the vaccinated groups, ranging from 5 to $11 \%, 16-36 / 294-315, p<0.05)$. when compared with the PBS control group 29\% (92/315) (Fig. 5). In addition, all groups receiving Ivag/topical imiquimod had reduced shedding rates $(5-6 \%)$ compared with the group receiving SQ imiquimod (11\%). Lastly, the shedding rate in the monovalent gD2 group+Ivag/topical imiquimod was similar to and in effect lower than the trivalent or bivalent groups+lvag/topical imiquimod (Fig. 5a). Importantly, none of the vaccinated groups shed detectable (infectious) virus when evaluated by culture compared with the PBS group where virus was recovered from $1.2 \%$ of the swabs. Evaluating shedding after the third immunization provided similar results as all the vaccinated groups had siginficanty $(p<$ 0.001) fewer positive swabs than the PBS group (Fig. 5b).

Further, the quantity of HSV-2 DNA in the swabs that were positive was lower in each of the Ivag/topical imiquimod groups

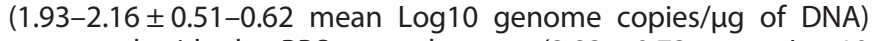
compared with the PBS control group $(2.83 \pm 0.73$ mean Log 10 genome copies/ug of DNA) (Fig. 5c). Two of the groups treated with Ivag/topical imiquimod (Trivalent and gD2 $+\mathrm{gE2}$ ) also had a reduction in the quantity of HSV-2 DNA $(2.04 \pm 0.62$ and $1.93 \pm$ 0.58 mean Log10 genome copies/Mg of DNA, respectively) when compared to the Trivalent group treated with SQ imiquimod

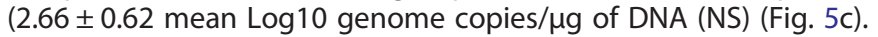

The number of animals with at least one positive swab sample in the study was also analyzed. The bivalent group gD2+gE2 and the monovalent gD2 group treated with Ivag/topical imiquimod had the most animals without a positive swab $(8 / 14,57 \%$ and $8 / 15,53 \%$, respectively) as compared to both the PBS control group $(0 / 15,0 \%)$ and the trivalent group treated with SQ imiquimod $0 / 15, p<0.05$ ).

Analysis of the frozen vaginal sections from experiment 2 again showed that groups administered vaccine+lvagl/topical imiquimod had an increase in both CD4 but predominately CD8 T cells when compared with placebo or the group given vaccine and SQ imiquimod. The animals vaccinated with $\mathrm{gC} 2 / \mathrm{gD} 2 / \mathrm{gE} 2+\mathrm{lvag} /$ topical imiquimod appear to have the most CD8 T cells (Fig. 6).

\section{DISCUSSION}

Reducing recurrent HSV disease using immunotherapy is a major goal for HSV vaccine developers. Recent efforts have used a bivalent HSV vaccine and adjuvant to achieve an $\sim 50 \%$ reduction in recurrent HSV disease and recurrent shedding. ${ }^{13,14}$ Although this demonstrates the proof of concept for a therapeutic vaccine it falls somewhat short when compared to newer antiviral therapies, which can reduce recurrent disease and shedding by almost $90 \%{ }^{16,17}$ Nevertheless, the advantages of vaccine strategies, requiring infrequent administration less than yearly vs. drugs that must be given daily suggest that improving vaccine effectiveness is a worthwhile endeavor.

Previous work has shown that there is a population of CD8 $T$ cells that can be found near nerve endings that control reactivated $\mathrm{HSV}^{18,19}$ Further, in simulations the local CD8 Tlymphocyte density in the mucosa predicted the peak HSV DNA copy number and whether genital lesions or subclinical shedding occurred..$^{20}$ Their model suggested that the rate of containment of 


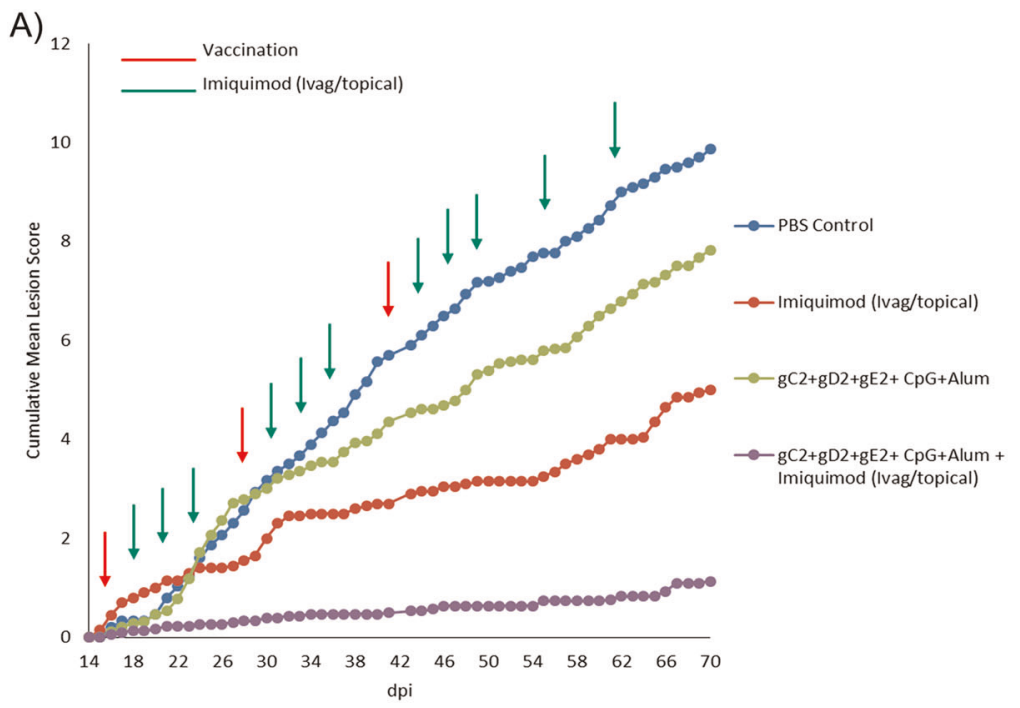

B)

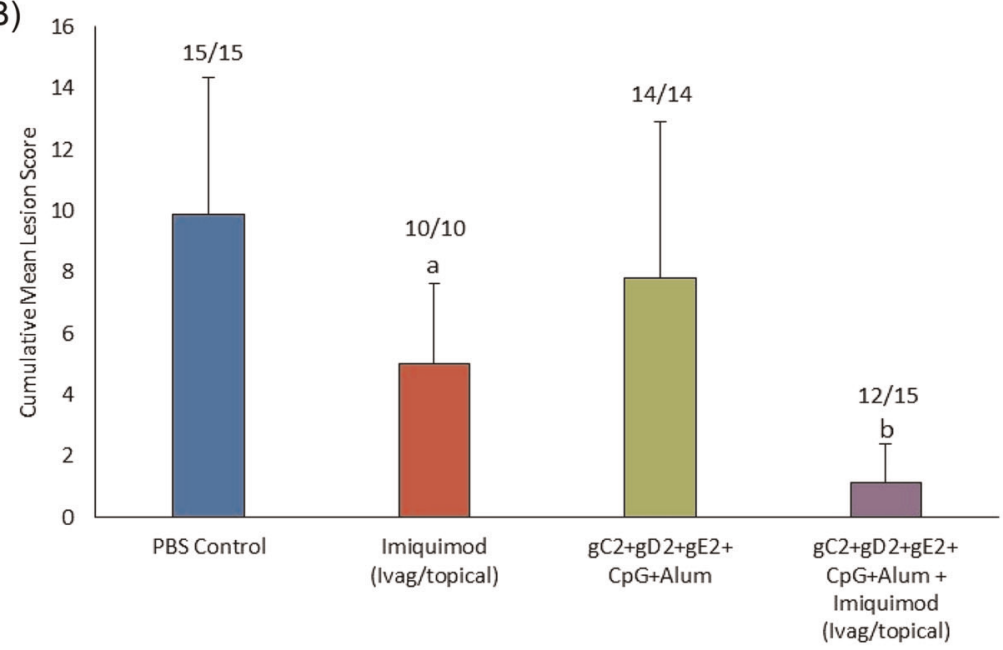

C)

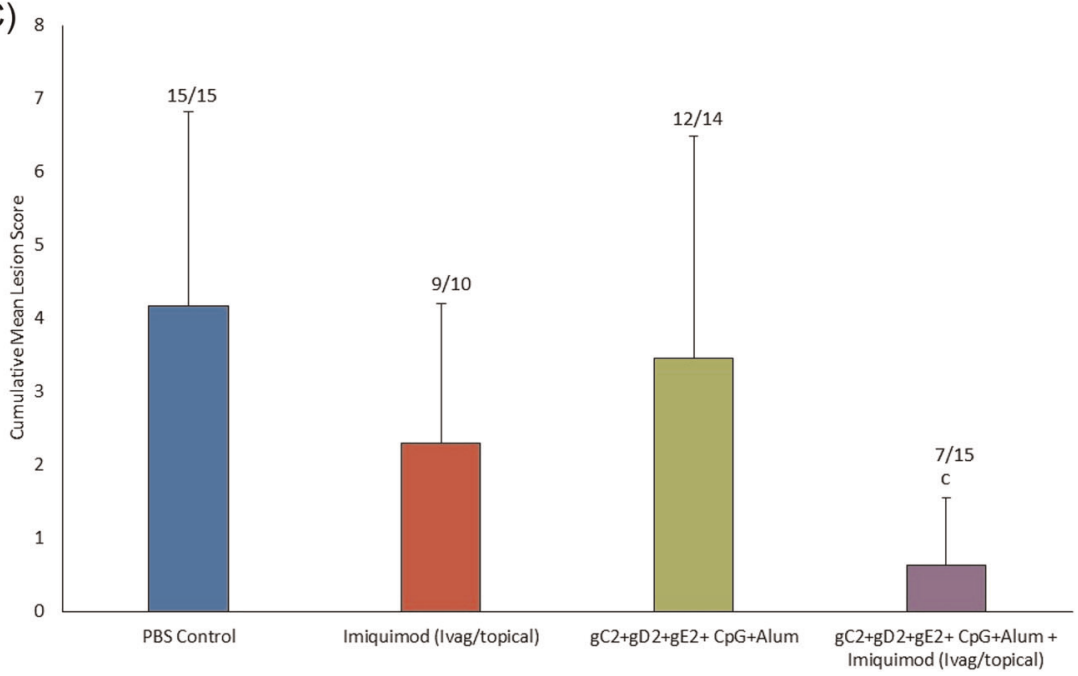

Fig. 1 Recurrent Lesion Scores. In study 1, HSV-2 infected animals were either vaccinated with trivalent vaccine, treated Ivag/topical with imiquimod, both or neither as described in the methods. Animals were then followed daily for recurrent lesions from day 15-70. a shows the cumulative recurrent lesion scores over the time period of day 15-70. b shows the total recurrent lesion score from the day after the first immunization (day 15) until day 70. c shows total recurrent lesion score from the day after the third immunization (day 43 ) until day 70 . The numbers above the bars are the number of animals with recurrent lesion/total number of animals ${ }^{\mathrm{a}} p<0.05$ vs $\mathrm{PBS}$; ${ }^{\mathrm{b}} p<0.001$ vs vaccine alone group ${ }^{\mathrm{C}} P<0.001$ vs. PBS and $P<0.01$ vs the vaccine alone group comparing mean lesion scores 

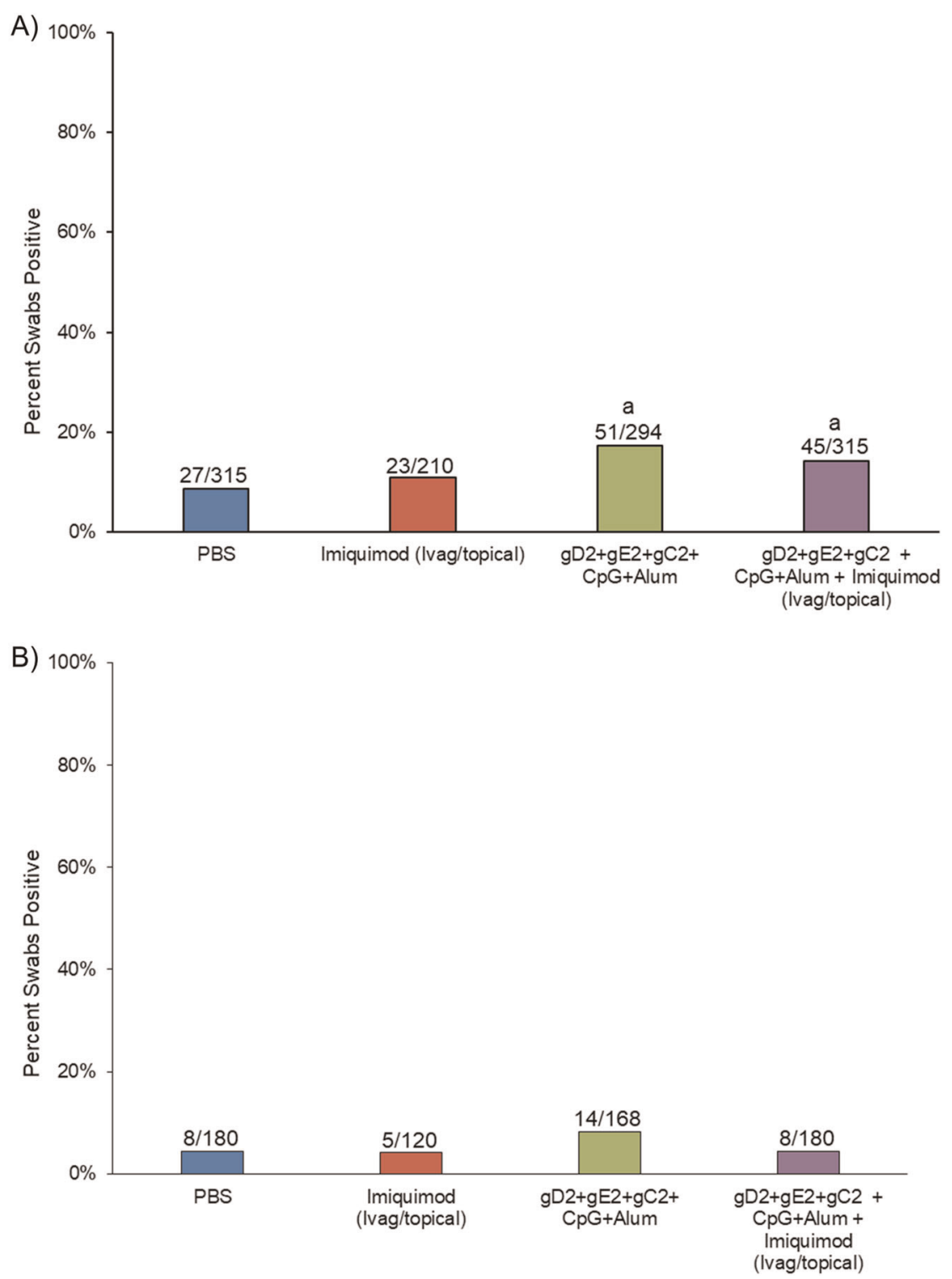

Fig. 2 Percent (number of recurrent swabs with detectable HSV DNA). In study 1, HSV-2 infected animals were either vaccinated with trivalent vaccine, treated Ivag/topical with imiquimod, both or neither as described in the methods. Vaginal swabs were then collected three times per week from day 21-70. Swabs were evaluated by qPCR for the presence of HSV DNA and the number of swabs that were positive/total number of swabs for each group is presented above the bars and plotted as the per cent positive. a shows the total number of positive swabs from the day after the first immunization (day 15 ) until day 70 . b shows the number of positive swabs the day after the third immunization (day 43 ) until day $70 .{ }^{\mathrm{a}} p<0.05$ vs placebo

infected cells by the peripheral mucosal immune system is the major driver of duration and severity of HSV-2 reactivation.

We therefore sought to utilize the prime pull strategy originally described for prophylactic HSV vaccines by Shin and Iwasaki ${ }^{15}$ to enhance the number of HSV specific T cells that would control reactivated HSV as it emerges from nerve endings to cause recurrent disease and recurrent virus shedding.

In the first study, the prime pull strategy significantly decreased recurrent HSV-2 disease compared with the prime $(\mathrm{gD}+\mathrm{gC}+\mathrm{gE}$ vaccine alone) or the pull (Ivag/topical imiquimod) alone but curiously, none of the treatments reduced recurrent vaginal shedding. In the second study, designed to compare the trivalent vaccine to a monovalent $\mathrm{gD}$ vaccine and systemic vs. Ivag/topical imiquimod, we found that any of the vaccines when given with Ivag/topical significantly reduced both recurrent disease and recurrent vaginal shedding. Importantly, none of the vaccinated animals shed detectable infectious virus (limit of detection five
PFU/swab) during the period of observation for recurrences. We also found the monovalent vaccine was as effective as the trivalent or bivalent vaccines in reducing both recurrent disease and recurrent vaginal shedding and that Ivag/topical imiquimod was significantly more effective than systemic imiquimod. This later finding strongly suggests that imiquimod was acting more as a $\mathrm{T}$ cell attractant rather than as an adjuvant. ${ }^{21}$ This hypothesis is further supported by the immunofluorescent analysis that shows the increase in local CD8 T cells in the Prime and Pull treated guinea pigs.

It is not clear why the effects on HSV shedding were different between the two studies but it should be noted that the rate of shedding in the PBS group in the first study was $<10 \%$, which is substantially less than we usually observe, and was less than in the second study (29\%), making it difficult to detect an effect of vaccinations. Further, in the past shedding has been more difficult to affect than lesion development in this model for both 


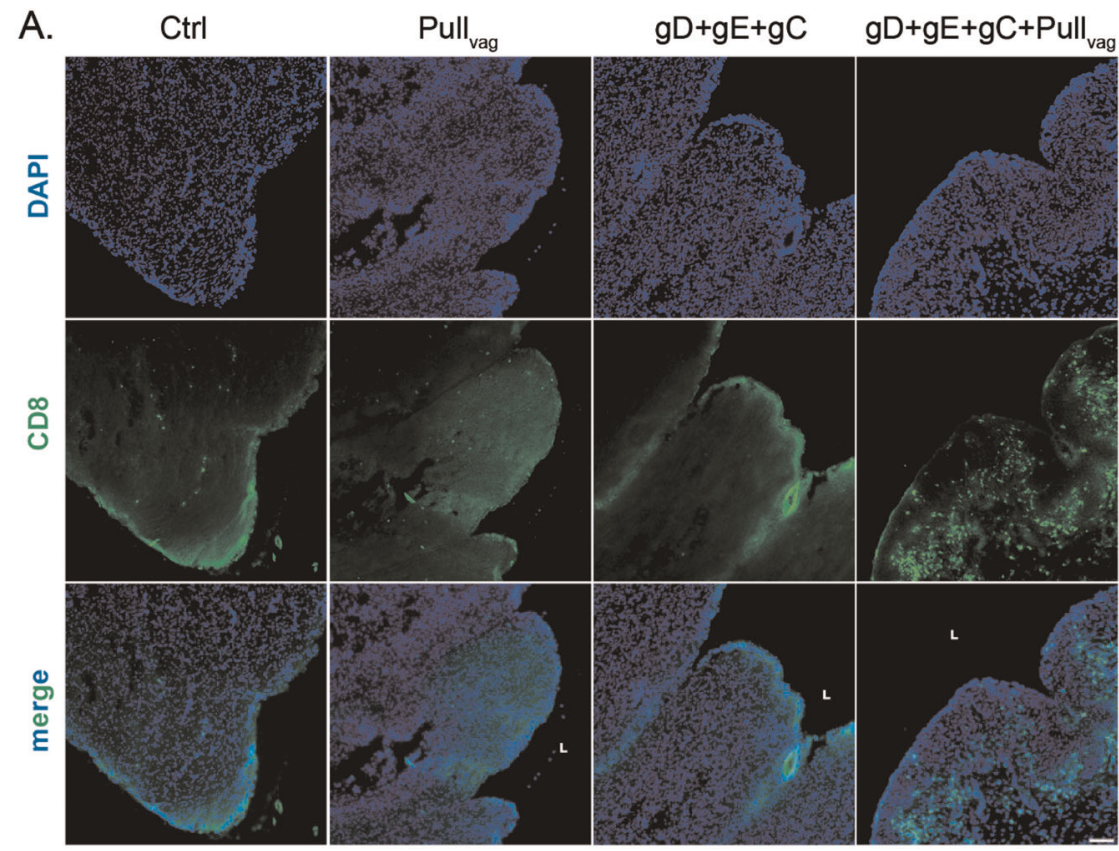

B.

Ctrl

Pull $_{\text {vag }}$

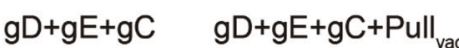

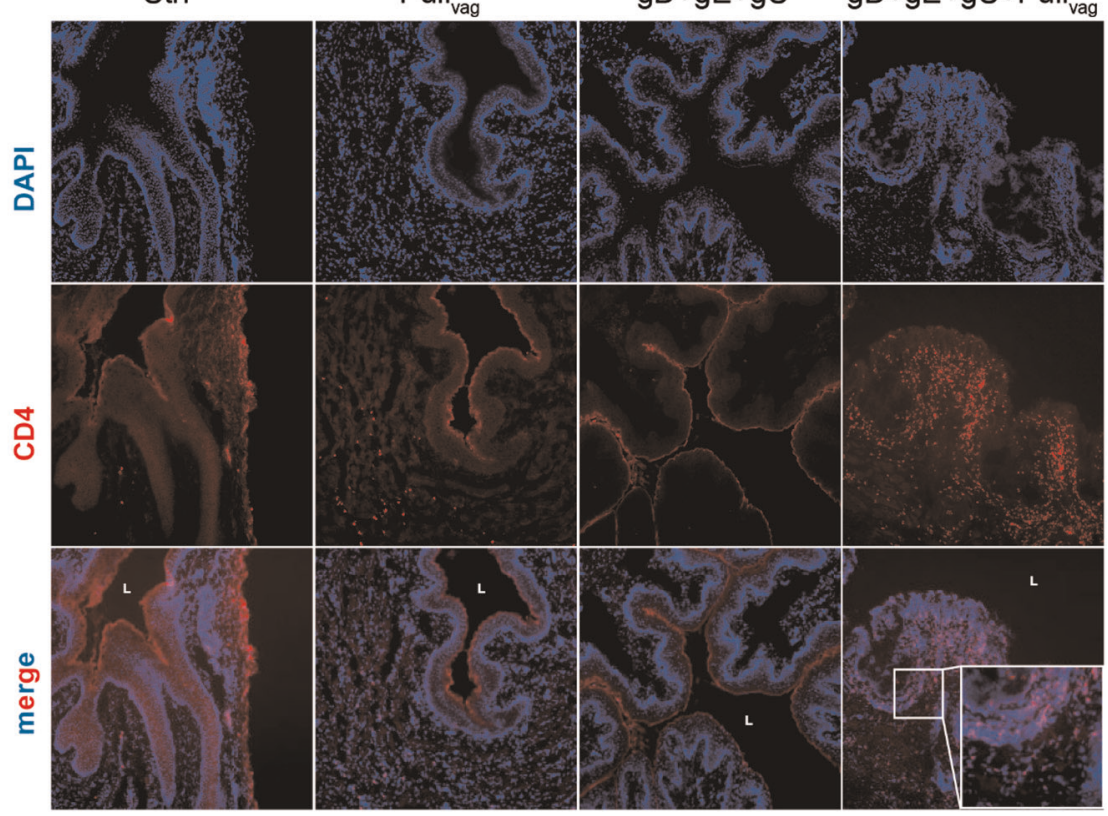

Fig. $3 \mathrm{CD} 4$ and CD8 T-cell localization in the vagina of guinea pigs that received trivalent vaccine Prime with or without Ivag/topical imiquimod Pull. Vaginas from four groups were collected at the termination of the study (day 70) and frozen in OCT. Frozen sections were stained with antibody to CD8 and CD4 and analyzed by fluorescence microscopy using the $\times 10$ objective lens. Scale represents $100 \mu \mathrm{m}$. a CD8 staining b CD4 staining. The $L$ notation marks the lumen of the vaginal cavity

antivirals $^{22}$ and vaccines. ${ }^{23,24}$ It is also possible that the small sample size and the variability in recurrent disease and shedding could result in inconsistent results. In human trials hundreds of subjects are often enrolled while animal studies usually use 10-20 animals/group.

Although no local irritation was noted in the guinea pigs, imiquimod may not have an acceptable safety profile for topical application in humans. Topical imiquimod creates a localized immune response that can produce signs of local irritation including erythema, edema, induration, ulcerations, and crusting ${ }^{25}$ with $\sim 10 \%$ of patients developing erosions and ulcerations at the application site. ${ }^{26}$ In addition, adverse reactions not only occur at the site of application, but also in the vicinity of application. ${ }^{27}$ For these reasons we are currently exploring other topical treatments, including lower doses of imiquimod, which would be less reactogenic but still enhance recruitment of $T$ cells. Although the primary pull response was for CD8 T cells, we detected an increase in CD4 T cells that potentially poses an added risk of HIV acquisition. The use of CD8-specific pull, such as recombinant chemokines CXCL9 and CXCL10, ${ }^{15}$ or agents that selectively elicit these chemokines may circumvent such concerns in the future.

In summary, using glycoprotein vaccines to boost HSV immunity and intravaginal/topical imiquimod to pull these immune cells into the vaginal tract significantly reduced recurrent 


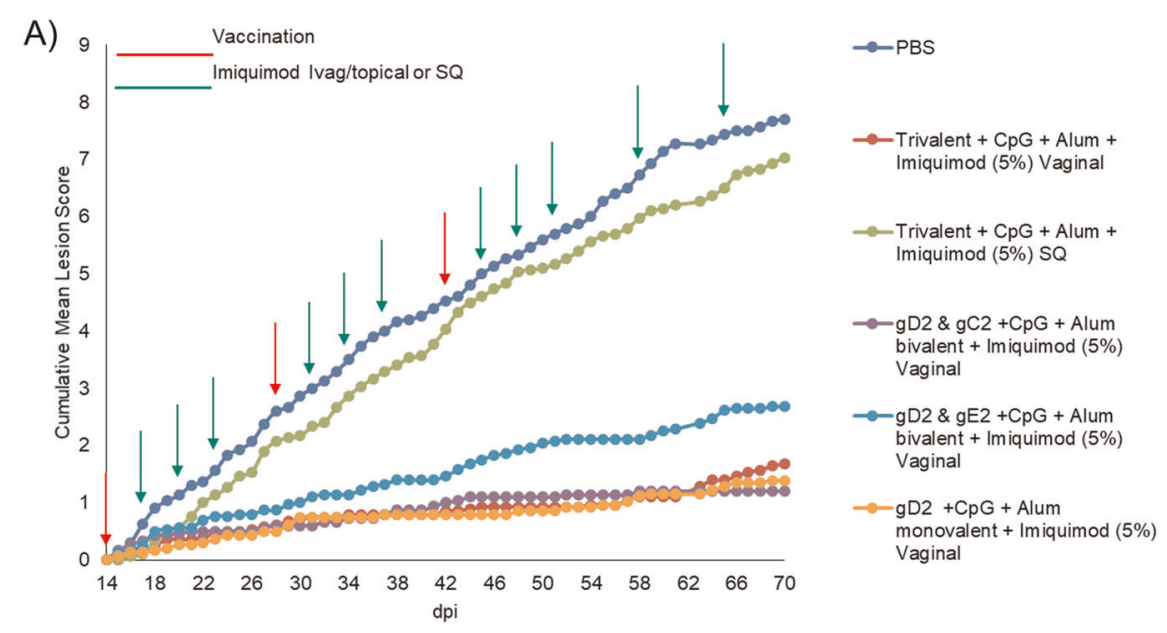

B)

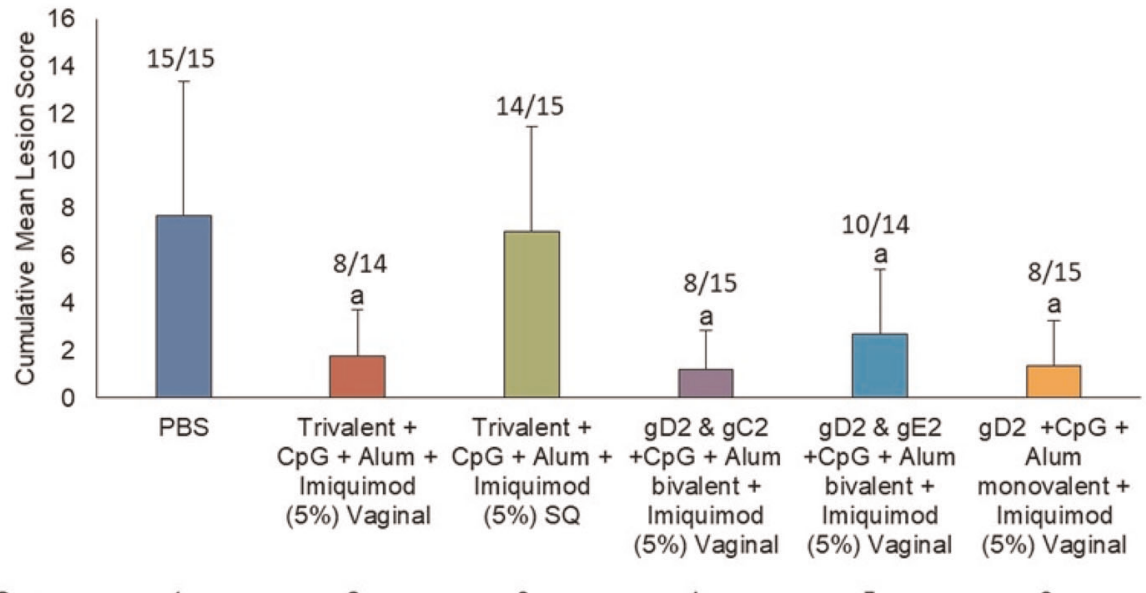

Group

1

2

3

4

5

C)

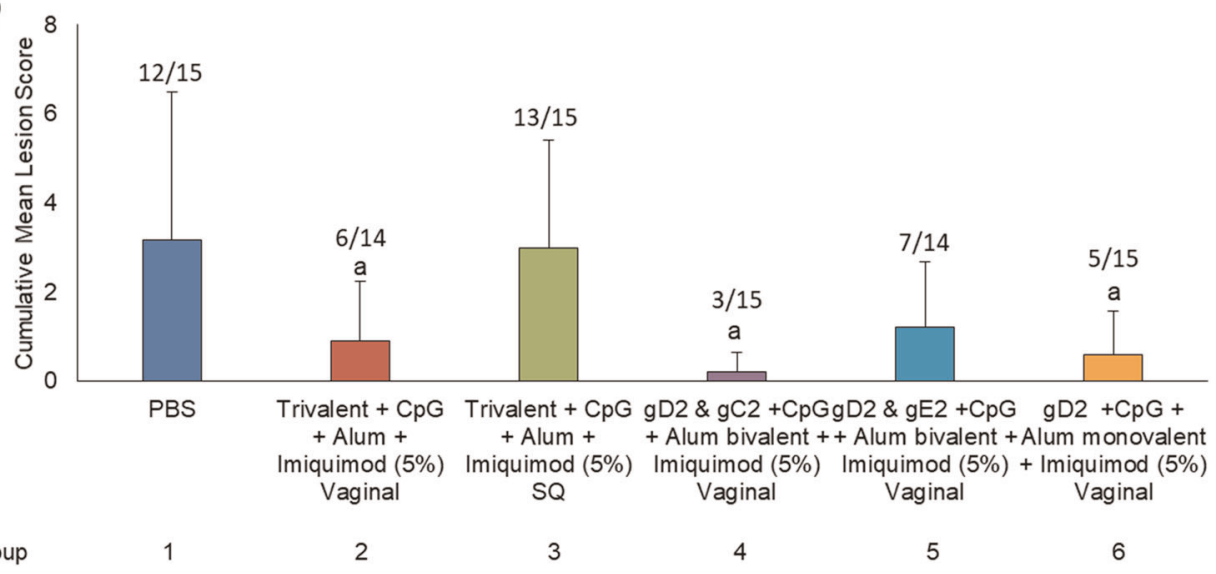

Fig. 4 Recurrent lesion scores for Study 2. In study 2, HSV-2 infected animals were either unvaccinated (PBS), vaccinated with trivalent vaccine and treated SQ with imiquimod, vaccinated with trivalent vaccine and treated Ivag/topical with imiquimod, vaccinated with bivalent or monovalent vaccine and treated Ivag/topical with imiquimod. Animals were then followed daily for recurrent lesions from day 15-70. a shows the cumulative recurrent lesion scores over the time period of day 15-70. b shows the total recurrent lesion score from the day after the first immunization (day 15) until day 70. c shows total recurrent lesion score from the day after the third immunization (day 43 ) until day 70 . The number above the bars shows the number of animals with recurrent lesions/ total number of animals. ${ }^{a} p \leq 0.05$ vs group 1 and 3

genital HSV lesions and the shedding of HSV-2 into the vaginal tract. These results reveal the effectiveness of the Prime and Pull vaccination strategy as it applies to therapeutic vaccines against genital herpes.

\section{METHODS}

\section{Vaccines}

The baculovirus-expressed gC2 protein bac-gC2(426t) extends from amino acid 27 to amino acid 426, where amino acid 27 is the first amino acid after the signal peptide. ${ }^{28,29}$ The baculovirus-expressed gD2 protein bac-gD2 

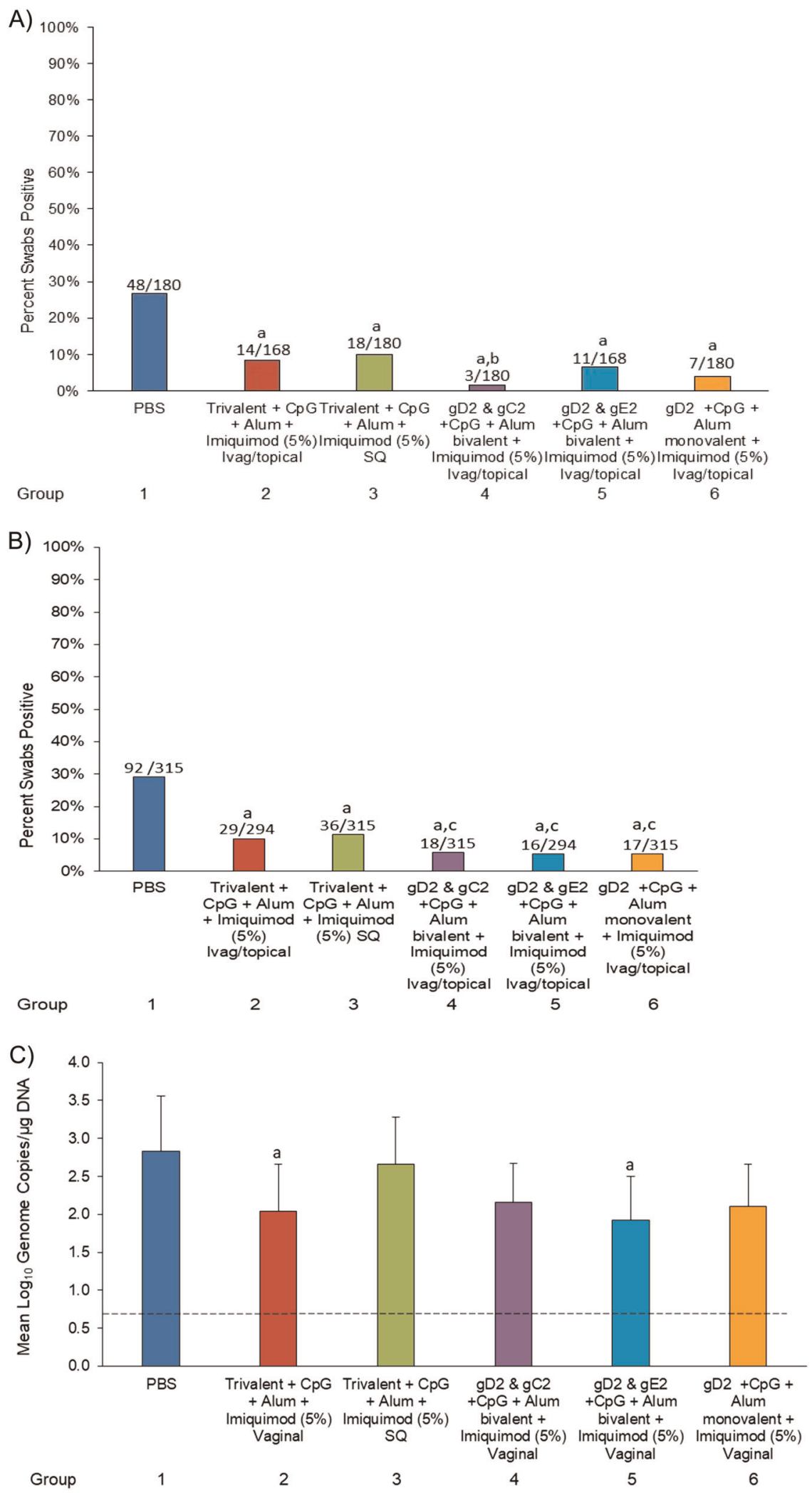

Fig. 5 Recurrent vaginal shedding. In study 2, HSV-2 infected animals were either unvaccinated, vaccinated with trivalent vaccine and treated SQ with imiquimod, vaccinated with trivalent vaccine and treated Ivag/topical with imiquimod, vaccinated with bivalent or monovalent vaccine, and treated with Ivag/topical imiquimod as described in the methods. Vaginal swabs were then collected three times per week from day 21-70. Swabs were evaluated by qPCR for the presence of HSV DNA. a shows the number of swabs that were positive/total number of swabs for each group as presented above the bars and plotted as the per cent positive for the entire period of swabbing (d21-70). $\mathbf{b}$ shows this information for the period after the third immunization (day 43-70). c shows the quantity of HSV-2 DNA in the positive swabs from day $21-70$ with the dashed line representing the limit of detection. ${ }^{a} p<0.001$ vs group $1 ;{ }^{b} p<0.05$ vs group $2{ }^{c} p<0.05$ vs. group 3 

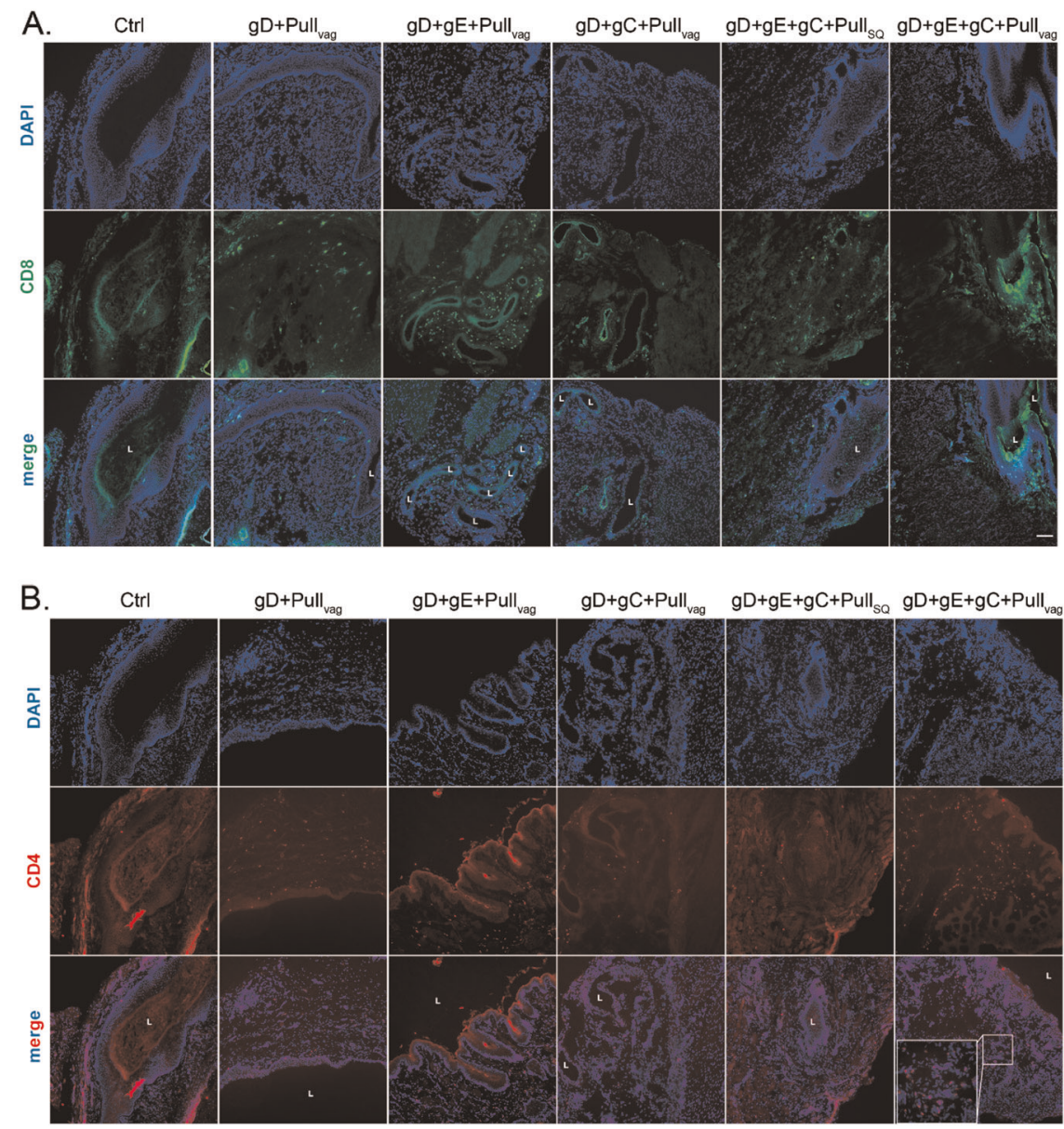

Fig. $6 \mathrm{CD} 4$ and CD8 T-cell localization in the vagina of guinea pigs that received monovalent ( $\mathrm{gD}$ ), bivalent ( $\mathrm{gD}+\mathrm{gC}$ or $\mathrm{gD}+\mathrm{gE})$, or trivalent $(\mathrm{gD}+\mathrm{gC}+\mathrm{gE})$ vaccine with either SQ imiquimod or Ivag/topical imiquimod. Vaginas were collected at the termination of the study (day 70 ) and frozen in OCT. Frozen sections were stained with antibody to CD8 and CD4 and analyzed by fluorescence microscopy using the $\times 10$ objective lens. Scale represents $100 \mu \mathrm{m}$. a CD8 staining b CD4 staining. The L notation marks the lumen of the vaginal cavity

(306t) extends from amino acid 26 to amino acid 331, where amino acid 26 is the first amino acid after the signal peptide. ${ }^{29-31}$ The methods used to construct bac-gD2(306t) resulted in an aspartic acid and a proline being added at the $\mathrm{N}$ terminus. The baculovirus-expressed gE2 protein bac-gE2 (24-405t) extends from gE2 amino acid 24 to 405 where amino acid 24 is the first amino acid after the signal peptide. ${ }^{32}$

Vaccines were administered at $10 \mu \mathrm{g}$ for each protein/animal intramuscularly in the quadriceps with a total volume of $50 \mu \mathrm{l}$ per immunization. ${ }^{29}$

\section{Adjuvant}

CpG oligonucleotide (5'-TCGTCGTTGTCGTITGTCGTT-3') (Trilink Inc.) was administered using $100 \mu \mathrm{g} \mathrm{CpG/guinea} \mathrm{pig.} \mathrm{Alum} \mathrm{(Alhydrogel,} \mathrm{Accurate}$ Chemical and Scientific Corp.) was used at $240 \mu \mathrm{g} /$ guinea pig.

\section{Imiquimod}

Aldara (imiquimod) $)^{21,33}$ was purchased from Cincinnati Children's Hospital pharmacy as a $5 \%$ cream and $0.1 \mathrm{ml}$ applied intravaginally using a $1 \mathrm{ml}$ syringe and $0.1 \mathrm{ml}$ applied to the external genital skin (area not covered by hair) When administered systemically it was purchased from Sigma and prepared with $5 \%$ dimethylsulfoxide in PBS and administered subcutaneously as $0.5 \mathrm{mg} / \mathrm{kg} / \mathrm{dose}$ in the upper shoulder (referred to as SQ).

\section{Study design}

In the first evaluation, 60 female Harley guinea pigs (250-300 gms, Charles River Breeding Laboratories (Wilmington, MA)) were obtained and housed under AAALAC approved conditions at Cincinnati Children's Hospital Medical Center. Animals were divided into the following four groups: $(N=$ 15/group):
1. PBS control

2. Imiquimod Ivag/topical

3. $\mathrm{gC2}+\mathrm{gD} 2+\mathrm{gE}+\mathrm{CpG}+$ Alum (trivalent vaccine)

4. $\mathrm{gC} 2+\mathrm{gD} 2+\mathrm{gE}+\mathrm{CpG}+$ Alum (trivalent vaccine)+imiquimod Ivag/ topical

In the second experiment, 90 animals as described above were divided into the following six groups: $(N=15 /$ group $)$

1. PBS control

2. $\mathrm{gC} 2+\mathrm{gD} 2+\mathrm{gE}+\mathrm{CpG}+$ Alum (trivalent vaccine)+imiquimod, Ivag/ topical

3. $\mathrm{gC2}+\mathrm{gD} 2+\mathrm{gE}+\mathrm{CpG}+$ Alum (trivalent vaccine) + imiquimod, $\mathrm{SQ}$

4. $\mathrm{gD} 2$ and $\mathrm{gC2}+\mathrm{CpG}+$ Alum (bivalent vaccine)+imiquimod, Ivag/ topical

5. gD2 and $\mathrm{gE2}+\mathrm{CpG}+$ Alum (bivalent vaccine)+imiquimod, Ivag/ topical

6. gD2+CpG+Alum (monovalent vaccine)+imiquimod, Ivag/topical

After HSV-2 infection, immunizations with subunit proteins were performed three times at 2-week intervals.

Animals were infected by intravaginal administration of $1 \times 10^{6} \mathrm{pfu}, \mathrm{HSV}$ 2 strain MS as previously described. ${ }^{34}$ After inoculation a vaginal swab was collected at 2 dpi to document infection. Only animals infected with HSV-2 were vaccinated. The vaccines contained $10 \mu \mathrm{g}$ of each of antigen/animal, and were mixed with CpG (100 $\mu$ g/animal) and $240 \mu \mathrm{g} / \mathrm{animal}$ of Alum. Vaccines were administered intramuscularly (IM) three times at 14,28 , and 42 days after infection. Imiquimod was applied topically, as described above, or administered SQ on days 3, 6, and 9 after each vaccinationand then administered once weekly beginning on day 16 after the last vaccine.

Animals were observed daily for evidence of local irritation and for recurrent lesions (days 15-70) and vaginal swabs were collected on 
Monday, Wednesday and Friday (days 21-70) for evaluation of virus shedding as previously described. ${ }^{35,36}$ Recurrences were assigned a value of 1.0 if a vesicle was observed and 0.5 if only a red lesion was observed. The mean lesion score was obtained by averaging the scores for each animal over the duration of observation. Cumulative lesion scores are the average of the scores over time

All animal investigations were approved by the Institutional Animal Care and Use Committee at CCHMC.

\section{Immunofluorescence analysis}

At the end of the study (70 dpi), the animals were sacrificed. Vaginal tract was excised and then frozen in OCT compound (Sakura Finetek USA). OCTembedded 8- $\mu \mathrm{m}$ cryostat sections mounted onto Superfrost Plus Slides (Electron Microscopy Sciences) were fixed with cold acetone before being blocked in $0.1 \mathrm{~m}$ Tris- $\mathrm{HCl}$ buffer with $1 \%$ fetal bovine serum. Phycoerythrinconjugated anti-CD8 antibody (clone: CT6, Bio-Rad) or PE-conjugated anti CD4 antibody (clone: CT7, Bio-Rad) staining was followed by a secondary antibody, Rhodamine conjugated anti-R Phycoerythrin (Abcam). Slides were stained with 4',6-diamidino-2-phenylindole (Sigma) and mounted with Prolong Gold Antifade reagent (Thermo fisher). All slides were analyzed by fluorescence microscopy (BX51; Olympus) with 10x lens. Imaging data were analyzed with Imaris 7.2 (Bitplane) and ImageJ $1.46 \mathrm{r}(\mathrm{NIH})$.

PCR

Vaginal swabs were stored at $-80^{\circ} \mathrm{C}$. DNA was isolated from $200 \mu \mathrm{l}$ of vaginal swab media using QIAamp DNA Mini Kit (Qiagen \#51306) according to manufacturer's protocol. To determine the amount of HSV2 shed, HSV-2 gG2 gene detection was performed by quantitative PCR. The gG2 primer and probe sequences were as followed: ${ }^{35}$ Forward: $5^{\prime}-\mathrm{CGG} /$ AGA/CAT/TCG/AGT/ACC/AGA/TC-3'; reverse: 5'- GCC/CAC/CTC/TAC/CCA/ CAA/CA-3'; and probe: 5'-FAM-ACC/CAC/GTG/CAG/CTC/GCC/G -tamRA-3'.

Each PCR reaction contained $100 \mathrm{ng}$ of DNA, $50 \mu \mathrm{m}$ of each primer, $0.10 \mu \mathrm{M}$ of FAM/tamRA fluorescent probe, and $10 \mu \mathrm{l}$ of Taqman Gene Expression Master Mix (ABI) in a total volume of $20 \mu \mathrm{l}$ reaction. PCR amplification of HSV-2 DNA was performed on a 7500 Fast Real-Time PCR system (ABI) using the following conditions: pre-incubation at $50^{\circ} \mathrm{C}$ for $2 \mathrm{~min}$ and $95^{\circ} \mathrm{C}$ for $10 \mathrm{~min}$ followed by 50 cycles consisting of a denaturation step at $95^{\circ} \mathrm{C}$ for $15 \mathrm{~s}$, annealing at $60^{\circ} \mathrm{C}$ for 1 minute, and elongation at $72^{\circ} \mathrm{C}$ for $10 \mathrm{~s}$. A standard curve for each virus was generated with ten-fold serial dilutions of purified HSV-2 DNA (ATCC) containing $10^{5}-10^{0} \mathrm{HSV}-2$ copies in $50 \mathrm{ng}$ of uninfected guinea pig brain DNA. The limit of detection for HSV-2 was determined to be between $10^{\circ}$ and $10^{1}$ copies, with excellent linearity $(R \geq 0.98)$ over five logs of HSV genomic DNA content.

Virus cultures from vaginal swabs

Frozen vaginal swabs were quickly thawed and swab media added to Vero cell monolayers in 24-well plates for 1 hour at $37^{\circ} \mathrm{C}$. Cells were overlaid with $0.5 \%$ methylcellulose in complete DMEM supplemented with $25 \mu \mathrm{g}$ vancomycin. ${ }^{37}$ Plaques were counted 5 days later. The lower limit of detection was $<5 \mathrm{PFU} / \mathrm{ml}$.

\section{Statistical analysis}

For comparison of the means, an analysis of variance was initially performed and if significant differences among all the groups were noted, a Tukey's test to adjust for multiple comparison was used. Data are presented as means and standard deviations. Incidence data were compared by Fisher's exact test. All comparisons are two-tailed with a $P$ value of $<0.05$ considered significant.

\section{Reporting summary}

Further information on research design is available in the Nature Research Reporting Summary linked to this article.

\section{DATA AVAILABILITY}

The data sets generated and/or analyzed during the current study are available from the corresponding author.

\section{ACKNOWLEDGEMENTS}

This work was supported by National Institute of Health Contract No. HHSN272201000008I (DIB) as well as 01Al104854 (HF), R01Al054359, and R01Al127429 (A.I.). A.I. is an Investigator of the Howard Hughes Medical Institute. P.L. is a recipient of The James Hudson Brown-Alexander Brown Coxe Postdoctoral Fellowship. We thank Gary Cohen for providing the gC2, gD2, and gE2 subunit antigens used in these studies.

\section{AUTHOR CONTRIBUTIONS}

D.B., R.C., A.I. and H.F. were involved in the conception, design, data acquisition, analysis, interpretation, drafting, or revising the article for intellectual content. F.B., S. A., P.Lu, D.P. and D.D., were involved in data acquisition, analysis, interpretation and helped drafting or revising the article for intellectual content and accuracy.

\section{ADDITIONAL INFORMATION}

Supplementary information accompanies the paper on the npj Vaccines website (https://doi.org/10.1038/s41541-019-0129-1).

Competing interests: DIB is a consultant to Merck and Genocea regarding herpes vaccines. He also serves on a DSMB for Moderna. Harvey Friedman and Sita Awasthi are named on patents that describe the use of multiple subunit glycoprotein antigens for HSV vaccines. All other authors declare no competing interests.

Publisher's note: Springer Nature remains neutral with regard to jurisdictional claims in published maps and institutional affiliations.

\section{REFERENCES}

1. Looker, K. J. et al. Global estimates of prevalent and incident herpes simplex virus type 2 infections in 2012. PLoS ONE 10, e114989 (2015).

2. Wald, A. et al. Reactivation of genital herpes simplex virus type 2 infection in asymptomatic seropositive persons. N. Engl. J. Med. 342, 844-850 (2000).

3. Tronstein, E. et al. Genital shedding of herpes simplex virus among symptomatic and asymptomatic persons with HSV-2 infection. JAMA 305, 1441-1449 (2011).

4. Rooney, J. F., Felser, J. M., Ostrove, J. M. \& Straus, S. E. Acquisition of genital herpes from an asymptomatic sexual partner. N. Engl. J. Med. 314, 1561-1564 (1986).

5. Whitley, R. Neonatal herpes simplex virus infection. Curr. Opin. Infect. Dis. 17 243-246 (2004)

6. James, S. H. \& Kimberlin, D. W. Neonatal Herpes Simplex Virus Infection. Infect. Dis. Clin. North Am. 29, 391-400 (2015).

7. Johnson, L. F. \& Lewis, D. A. The effect of genital tract infections on HIV-1 shedding in the genital tract: a systematic review and meta-analysis. Sex. Transm. Dis. 35, 946-959 (2008).

8. Birkmann, A. \& Zimmermann, H. HSV antivirals-current and future treatment options. Curr. Opin. Virol. 18, 9-13 (2016).

9. Hollier, L. M. \& Eppes, C. Genital herpes: oral antiviral treatments. BMJ Clin. Evid. 2015, 1603 (2015).

10. Cohen, J. I. Vaccination to reduce reactivation of herpes simplex virus type 2 . J. Infect. Dis. 215, 844-846 (2017).

11. Stanberry, L. R. et al. Herpes simplex virus glycoprotein immunotherapy of recurrent genital herpes: factors influencing efficacy. Antivir. Res. 11, 203-214 (1989).

12. Myers, M. G., Bernstein, D. I., Harrison, C. J. \& Stanberry, L. R. Herpes simplex virus glycoprotein treatment of recurrent genital herpes reduces cervicovaginal virus shedding in guinea pigs. Antivir. Res. 10, 83-88 (1988).

13. Bernstein, D. I. et al. Therapeutic vaccine for genital herpes simplex virus-2 infection: findings from a randomized trial. J. Infect. Dis. 215, 856-864 (2017).

14. Van Wagoner, N. et al. Effects of different doses of GEN-003, a therapeutic vaccine for genital herpes simplex virus-2, on viral shedding and lesions: results of a randomized placebo-controlled trial. J. Infect. Dis. 218, 1890-1899 (2018).

15. Shin, H. \& Iwasaki, A. A vaccine strategy that protects against genital herpes by establishing local memory T cells. Nature 491, 463-467 (2012).

16. Wald, A. et al. Effect of pritelivir compared with valacyclovir on genital HSV-2 shedding in patients with frequent recurrences: a randomized clinical trial. JAMA 316, 2495-2503 (2016)

17. Wald, A. et al. Helicase-primase inhibitor pritelivir for HSV-2 infection. N. Engl. J. Med. 370, 201-210 (2014).

18. Zhu, J. et al. Virus-specific CD8+ T cells accumulate near sensory nerve endings in genital skin during subclinical HSV-2 reactivation. J. Exp. Med. 204, 595-603 (2007). 
19. Zhu, J. et al. Immune surveillance by CD8alphaalpha+ skin-resident $T$ cells in human herpes virus infection. Nature 497, 494-497 (2013).

20. Schiffer, J. T. et al. Mucosal host immune response predicts the severity and duration of herpes simplex virus-2 genital tract shedding episodes. Proc. Natl Acad. Sci. USA 107, 18973-18978 (2010).

21. Bernstein, D. I., Harrison, C. J., Tepe, E. R., Shahwan, A. \& Miller, R. L. Effect of imiquimod as an adjuvant for immunotherapy of genital HSV in guinea-pigs. Vaccine 13, 72-76 (1995).

22. Bernstein, D. I. et al. Efficacy of N-methanocarbathymidine against genital herpes simplex virus type 2 shedding and infection in guinea pigs. Antivir. Chem. Chemother. 24, 19-27 (2015).

23. Bernstein, D. I. et al. Effects of herpes simplex virus type 2 glycoprotein vaccines and CLDC adjuvant on genital herpes infection in the guinea pig. Vaccine 29, 2071-2078 (2011).

24. Bourne, N. et al. Herpes simplex virus (HSV) type 2 glycoprotein D subunit vaccines and protection against genital HSV-1 or HSV-2 disease in guinea pigs. $J$. Infect. Dis. 187, 542-549 (2003).

25. Al-Mutairi, N., Al-Doukhi, A., Al-Farag, S. \& Al-Haddad, A. Comparative study on the efficacy, safety, and acceptability of imiquimod $5 \%$ cream versus cryotherapy for molluscum contagiosum in children. Pediatr. Dermatol. 27, 388-394 (2010).

26. Campanelli, A. \& Lubbe, J. Erosive cheilitis after facial application of imiquimod 5\% cream. J. Eur. Acad. Dermatol. Venereol. 21, 1429-1430 (2007).

27. Chakrabarty, A. K., Mraz, S., Geisse, J. K. \& Anderson, N. J. Aphthous ulcers associated with imiquimod and the treatment of actinic cheilitis. J. Am. Acad. Dermatol. 52, 35-37 (2005).

28. Tal-Singer, R. et al. Interaction of herpes simplex virus glycoprotein gC with mammalian cell surface molecules. J. Virol. 69, 4471-4483 (1995).

29. Awasthi, S. et al. Immunization with a vaccine combining herpes simplex virus 2 (HSV-2) glycoprotein $\mathrm{C}(\mathrm{gC})$ and $\mathrm{gD}$ subunits improves the protection of dorsal root ganglia in mice and reduces the frequency of recurrent vaginal shedding of HSV-2 DNA in guinea pigs compared to immunization with gD alone. J. Virol. 85, 10472-10486 (2011).

30. Canziani, G. et al. Exploring biomolecular recognition using optical biosensors. Methods 19, 253-269 (1999).

31. Tengvall, S., Lundqvist, A., Eisenberg, R. J., Cohen, G. H. \& Harandi, A. M. Mucosal administration of CpG oligodeoxynucleotide elicits strong CC and CXC chemokine responses in the vagina and serves as a potent Th1-tilting adjuvant for recombinant gD2 protein vaccination against genital herpes. J. Virol. 80, 5283-5291 (2006)

32. Awasthi, S., Huang, J., Shaw, C. \& Friedman, H. M. Blocking herpes simplex virus 2 glycoprotein $\mathrm{E}$ immune evasion as an approach to enhance efficacy of a trivalent subunit antigen vaccine for genital herpes. J. Virol. 88, 8421-8432 (2014).

33. Miller, R. L., Tomai, M. A., Harrison, C. J. \& Bernstein, D. I. Immunomodulation as a treatment strategy for genital herpes: review of the evidence. Int. Immunopharmacol. 2, 443-451 (2002).

34. Stanberry, L. R., Bernstein, D. I., Burke, R. L., Pachl, C. \& Myers, M. G. Vaccination with recombinant herpes simplex virus glycoproteins: protection against initial and recurrent genital herpes. J. Infect. Dis. 155, 914-920 (1987).

35. Bernstein, D. I. et al. The adjuvant CLDC increases protection of a herpes simplex type 2 glycoprotein D vaccine in guinea pigs. Vaccine 28, 3748-3753 (2010).

36. Awasthi, S., Hook, L. M., Shaw, C. E. \& Friedman, H. M. A trivalent subunit antigen glycoprotein vaccine as immunotherapy for genital herpes in the guinea pig genital infection model. Hum. Vaccin Immunother. 13, 2785-2793 (2017).

37. Awasthi, S. et al. An HSV-2 trivalent vaccine is immunogenic in rhesus macaques and highly efficacious in guinea pigs. PLoS Pathog. 13, e1006141 (2017).

(C) (i)

Open Access This article is licensed under a Creative Commons Attribution 4.0 International License, which permits use, sharing, adaptation, distribution and reproduction in any medium or format, as long as you give appropriate credit to the original author(s) and the source, provide a link to the Creative Commons license, and indicate if changes were made. The images or other third party material in this article are included in the article's Creative Commons license, unless indicated otherwise in a credit line to the material. If material is not included in the article's Creative Commons license and your intended use is not permitted by statutory regulation or exceeds the permitted use, you will need to obtain permission directly from the copyright holder. To view a copy of this license, visit http://creativecommons. org/licenses/by/4.0/.

(c) The Author(s) 2019 